\title{
Application of Entropy Generation to Improve Heat Transfer of Heat Sinks in Electric Machines
}

\author{
Toni Eger ${ }^{1,2, *}$, Thomas Bol ${ }^{1}$, Ayothi Ramanathan Thanu ${ }^{3}$, László Daróczy ${ }^{2}$, Gábor Janiga ${ }^{2}$, \\ Rüdiger Schroth ${ }^{1}$ and Dominique Thévenin ${ }^{2}$ \\ 1 Robert Bosch SG GmbH, Robert-Bosch Strasse 2, 71701 Schwieberdingen, Germany; \\ thomas.bol@de.bosch.com (T.B.); ruediger.schroth@de.bosch.com (R.S.) \\ 2 Laboratory of Fluid Dynamics and Technical Flows, Otto von Guericke University Magdeburg, \\ Universitätsplatz 2, 39106 Magdeburg, Germany; laszlo.daroczy@ovgu.de (L.D.); \\ gabor.janiga@ovgu.de (G.J.); thevenin@ovgu.de (D.T.) \\ 3 Robert Bosch Engineering and Business Solutions Limited, CHIL-SEZ Keeranatham Village, \\ 641035 Tamil Nadu Coimbatore, India; ayothiramanathan.thanu@in.bosch.com \\ * Correspondence: toni.eger@de.bosch.com; Tel.: +49-7062-911-8496 \\ Academic Editor: Yan Jin \\ Received: 15 April 2017; Accepted: 26 May 2017; Published: 2 June 2017
}

\begin{abstract}
To intensify heat transfer within the complex three-dimensional flow field found in technical devices, all relevant transport phenomena have to be taken into account. In this work, a generic procedure based on a detailed analysis of entropy generation is developed to improve heat sinks found in electric machines. It enables a simultaneous consideration of temperature and velocity distributions, lumped into a single, scalar value, which can be used to directly identify regions with a high potential for heat transfer improvement. By analyzing the resulting entropy fields, it is demonstrated that the improved design obtained by this procedure is noticeably better, compared to those obtained with a classical analysis considering separately temperature and velocity distributions. This opens the door for an efficient, computer-based optimization of heat transfer in real applications.
\end{abstract}

Keywords: entropy generation; irreversibility ratio; second law analysis; optimization; electric machines

\section{Introduction}

To analyze and improve heat transfer in real devices, e.g., electric machines, the complete flow field has to be analyzed, since it controls energy transport processes and thus heat exchange. In many cases, the local flow properties vary widely, perhaps even encountering transition from laminar to turbulent conditions, which result in a complex 3D flow field. Identifying opportunities to improve heat transfer requires the analysis of an exceptionally large quantity of data after carrying out the corresponding simulations by Computational Fluid Dynamics (CFD). As the trend is moving towards shorter time-to-market periods for products, the development process must be accelerated in order to reach the best design as early as possible. For this purpose, CFD-based Optimization (also called CFD-O) is a promising approach, as discussed in [1]. However, the high complexity of alternator systems leads to long computing times and involves a large number of design parameters. Therefore, a very high amount of numerical effort is required for CFD-O. To make this possible, efficient solutions must be designed at each step (automatic design generation, application-driven parameters, efficient optimization techniques, revealing objectives).

The alternator itself consists of a rotor and stator domain, each of which possesses different components. During operation, the rotor domain is in motion and the fan blades create a pressure gradient which drives the flow. Air from the engine bay streams through the alternator, passes over the heat sink, and finally leaves the system in a radial direction. The stator domain includes, in particular, 
electronic components that are glued onto a heat sink to increase the wetted area and thus improve heat transfer. To enable the first aerodynamic studies and to identify the parameters controlling heat transfer, a reduced alternator model (called V-Channel in this work) has been proposed. It only covers the stator domain (the one important for the temperature of the electronic components), as shown in Figure 1.
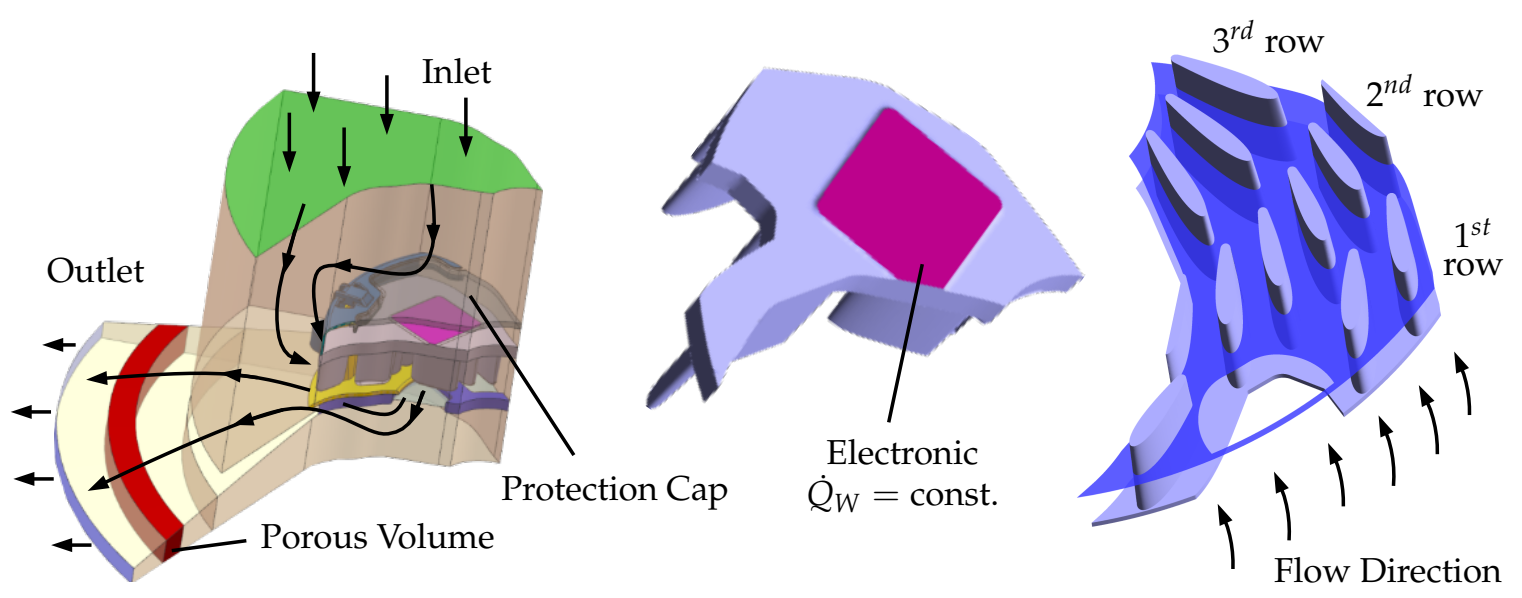

Figure 1. V-Channel setup employed to calculate heat transfer of heat sinks in electric machines. The dark blue color denotes the section plane located at mid-height of the National Advisory Committee for Aeronautics (NACA) profiles later used for the analysis of different indicators.

Inlet and outlet are associated in CFD to a constant-pressure boundary condition. For the side, periodic boundary conditions are employed in order to mimic the whole system, which is shown later in this article.

The rotor is not directly taken into account. The effect of the fans is represented by its characteristic curve, associated with the quadratic resistance coefficient $C_{R 2}$ resulting from the porous volume artificially placed before the outlet. Based on the real characteristic curve of the full model, a correct $\left(\Delta p, C_{R 2}\right)$-combination is determined for the rotational speed of each fan and is implemented into the CFD to obtain the correct operating point for all process conditions.

At this point, aerodynamic studies can be performed with a strongly reduced numerical effort, while still delivering results relevant for the full system. However, the V-Channel still involves too many design parameters for "brute force" CFD-O. In particular, a suitable indicator is needed to detect which regions within the immense data set describing the three-dimensional flow field are of central importance. Focusing only on these small target regions, analyzing design modifications to improve heat transfer then becomes possible. In this manner, the numerical effort can be suitably reduced, allowing CFD-O, as shown in this work.

As discussed in previous publications [2,3], different transport phenomena influence heat transfer. They can, in principle, be quantified by considering classical indicators such as the local wall temperature $T_{W}$ or the wall heat transfer coefficient $h$ for each component. However, these values show only the final outcome, at the very end of all heat transfer processes; they offer no further information on the underlying three-dimensional transport phenomena, which would be required to determine the factors limiting heat transfer and where or why these occur. Regions with a high potential for optimization cannot be directly obtained in this manner. Thus, physically-based indicators must first be identified, building on top of our previous studies [4,5].

\section{Entropy Generation}

The seminal work of Bejan [6-8] explains how the concept of entropy generation minimization (EGM) can be used to intensify heat transfer for different applications. Poulikakos and Bejan [9] derived 
the theoretical framework for an optimal fin geometry in forced convection using EGM. Fowler and Bejan [10] obtained optimal sizes of bodies for external flows. Carrington and Sun [11] analyzed internal and external flows with the second law analysis (SLA). Ko and Ting [12] investigated the competition between the entropy generation due to dissipation and conduction for laminar forced convection in curved rectangular ducts with external heating. Şahin [13] varied duct geometries, gave an analytical formulation and estimated the best geometry based on EGM. EGM in combination with genetic algorithms for optimization is described in [14,15], for example. Herwig et al. [16-18] extended the second law analysis and showed the potential of this approach for a variety of configurations. By additionally considering the fluctuating entropy generation terms, Kock and Herwig $[19,20]$ opened the door for advanced numerical investigations. Giangaspero and Sciubba $[21,22]$ used entropy generation to study thermal management for different electric machines. Several authors [23-26] have already successfully employed EGM to improve heat sink geometry. Note that a complete overview about the entropy generation minimization (EGM) method can be found in, e.g., [8] or [27]. The entropy generation is given by:

$$
\begin{aligned}
\dot{S}_{G e n} & =\int_{V}\left(\dot{S}_{\bar{C}}^{\prime \prime \prime}+\dot{S}_{C^{\prime}}^{\prime \prime \prime}+\dot{S}_{\bar{D}}^{\prime \prime \prime}+\dot{S}_{D^{\prime}}^{\prime \prime \prime}\right) \mathrm{d} V \\
& =\int_{V}(\underbrace{\frac{k}{\bar{T}^{2}}\left[\left(\frac{\partial \bar{T}}{\partial x}\right)^{2}+\left(\frac{\partial \bar{T}}{\partial y}\right)^{2}+\left(\frac{\partial \bar{T}}{\partial z}\right)^{2}\right]}_{\dot{S}_{\bar{C}}^{\prime \prime \prime}}+\underbrace{\frac{k}{\bar{T}^{2}} \frac{k_{\epsilon} \rho c_{p}}{\operatorname{Pr}_{t} \omega_{t} k}\left[\left(\frac{\partial \bar{T}}{\partial x}\right)^{2}+\left(\frac{\partial \bar{T}}{\partial y}\right)^{2}+\left(\frac{\partial \bar{T}}{\partial z}\right)^{2}\right]}_{\dot{S}_{C_{\bar{D}}^{\prime \prime \prime}}^{\prime \prime \prime}} \\
& +\underbrace{\frac{\mu}{\bar{T}}\left[2\left\{\left(\frac{\partial \bar{u}}{\partial x}\right)^{2}+\left(\frac{\partial \bar{v}}{\partial y}\right)^{2}+\left(\frac{\partial \bar{w}}{\partial z}\right)^{2}\right\}+\left(\frac{\partial \bar{u}}{\partial y}+\frac{\partial \bar{v}}{\partial x}\right)^{2}+\left(\frac{\partial \bar{u}}{\partial z}+\frac{\partial \bar{w}}{\partial x}\right)^{2}+\left(\frac{\partial \bar{v}}{\partial z}+\frac{\partial \bar{w}}{\partial y}\right)^{2}\right]} \\
& +\underbrace{\beta \frac{\rho \omega_{t} k_{e}}{\bar{T}}}_{\dot{S}_{D^{\prime}}^{\prime \prime \prime}}) \mathrm{d} V,
\end{aligned}
$$

with the time mean $(\bar{\square})$ and fluctuating $\left(\square^{\prime}\right)$ components for entropy generation due to conduction $\left(\dot{S}_{C}\right)$ and entropy generation due to dissipation $\left(\dot{S}_{D}\right)$, respectively. Thus, Equation (1) includes the temperature and velocity gradients, respectively, and represents the direct entropy generation in the mean and fluctuating flow field. A more detailed explanation of each term was given by Kock [28] and can also be found in Eger et al. [2]. The irreversibility ratio $\phi$ was first introduced by Bejan [29] and is defined as:

$$
\phi=\frac{\text { Dissipation }}{\text { Conduction }}=\frac{\dot{S}_{\bar{D}}^{\prime \prime \prime}+\dot{S}_{D^{\prime}}^{\prime \prime \prime}}{\dot{S}_{\bar{C}}^{\prime \prime \prime}+\dot{S}_{C^{\prime}}^{\prime \prime \prime}}=\frac{\dot{S}_{D}^{\prime \prime \prime}}{\dot{S}_{C}^{\prime \prime \prime}} .
$$

Considering total entropy generation $\dot{S}_{G e n}$ in the flow field enables a much deeper understanding of all processes controlling heat transfer. Compared to classical indicators such as $T$ or $h$, entropy generation quantifies regions of dominating processes between near-wall regions (locally) or within the main flow (globally), as well as dominating dissipative or conductive effects within the flow field. This has already been demonstrated in [2]. It also lumps the huge data quantity contained in $\mathbf{u}=\left(u_{x}, u_{y}, u_{z}\right)^{T}$ as well as temperature $T$ into one single, scalar value. Therefore, the amount of data that must be analyzed is strongly reduced, allowing systematic aerodynamic studies. Since it is defined as a second-law criterion, it is directly influenced by the properties and temperature levels of the fluid.

In a previous study [3], the generality of the irreversibility ratio $\phi$ in analyzing and improving heat transfer processes has been demonstrated for the simple canonical configuration first introduced 
in [4]. Based on $\phi$, regions with a high potential for optimization have been identified directly, in particular back-flow areas [3]. The design optimization automatically modified the sleeve in order to prevent areas with high values of $\phi$, leading to considerable intensification of heat transfer. Regions with $\phi \rightarrow \infty$ are easily identified, even in a complex three-dimensional flow field, and are promising for heat transfer improvement. Based on this observation, the irreversibility ratio will be considered, as well, in the present work in analyzing and optimizing a real application, which is far more complex than the canonical configuration considered in [3].

\section{Numerical Method}

The flow is calculated as stationary, considering a single-phase, non-reacting turbulent flow. Power losses on the electronic component are set to a constant value $\left(\dot{Q}_{W}=\right.$ const.). Due to the small temperature change in the flow field (approximately $20 \mathrm{~K}$ in this study), the working fluid is considered as an ideal gas with constant thermo-physical properties. For the defined ambient pressure of $p_{\infty}=1$ bar, the compressibility factor $Z$ is defined with 1.0000 [30], which ensures that this equation of state is fully appropriate for the current study. The equations of conservation of mass, momentum, and energy are discretized in ANSYS CFX 16.2, relying on the Reynolds-averaged Navier-Stokes equations (RANS) approach. Menter's $k$ - $\omega$-SST model has been chosen due to its ability to account for the low Reynolds regime near the wall without using damping corrections; in addition, it leads to an improved prediction of flow separation [31]. The selected advection model uses a second-order scheme wherever possible and blends to a first-order scheme, if needed, to maintain boundedness. The flow equations are solved sequentially with double precision.

\section{Grid-Independence Test}

To ensure that the discretization error is in an acceptable range, a grid-independence test was first performed. Varying the parameters controlling the maximum cell size $\left(s_{\max }\right)$ as well as the near-wall cell size $\left(s_{12}\right)$ leads to four different grids of increasing dimension, with up to almost 9 million grid points. Figure 2 shows the selected grid for the upcoming studies with approximately $4.8 \times 10^{6}$ nodes.

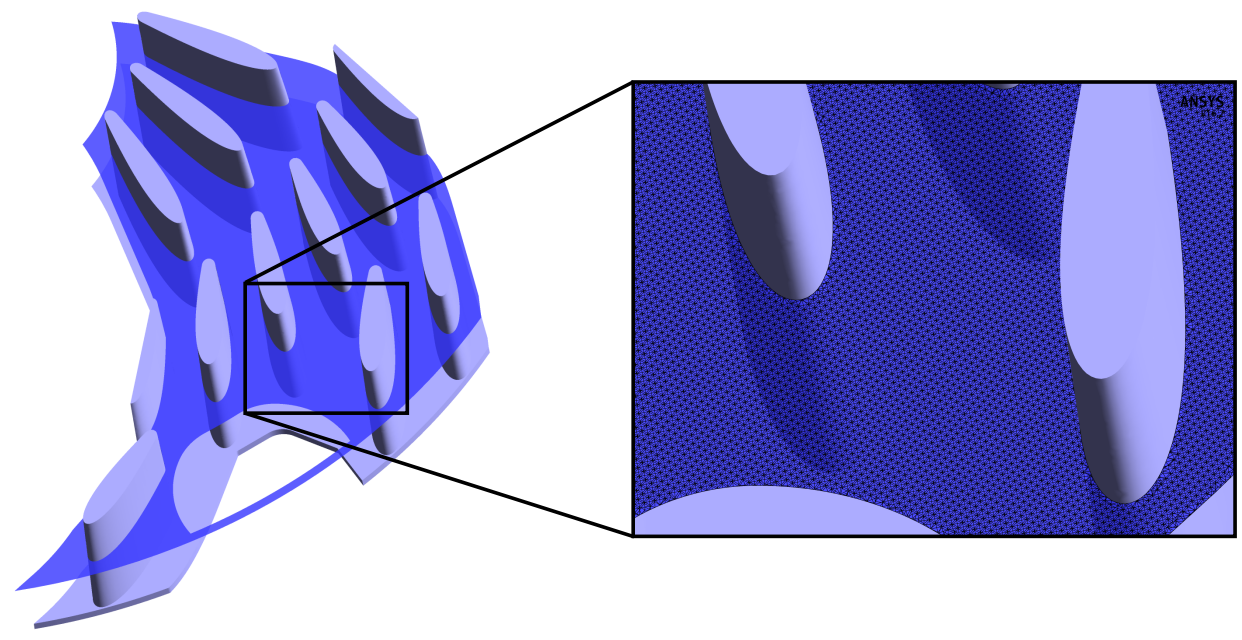

Figure 2. Section plane and selected grid later used for the analysis within the V-Channel.

The behavior of the dimensionless temperature difference:

$$
\Theta=\left(\frac{T_{W}-T_{\infty}}{T_{\infty}}\right)
$$

is shown in Figure 3. 

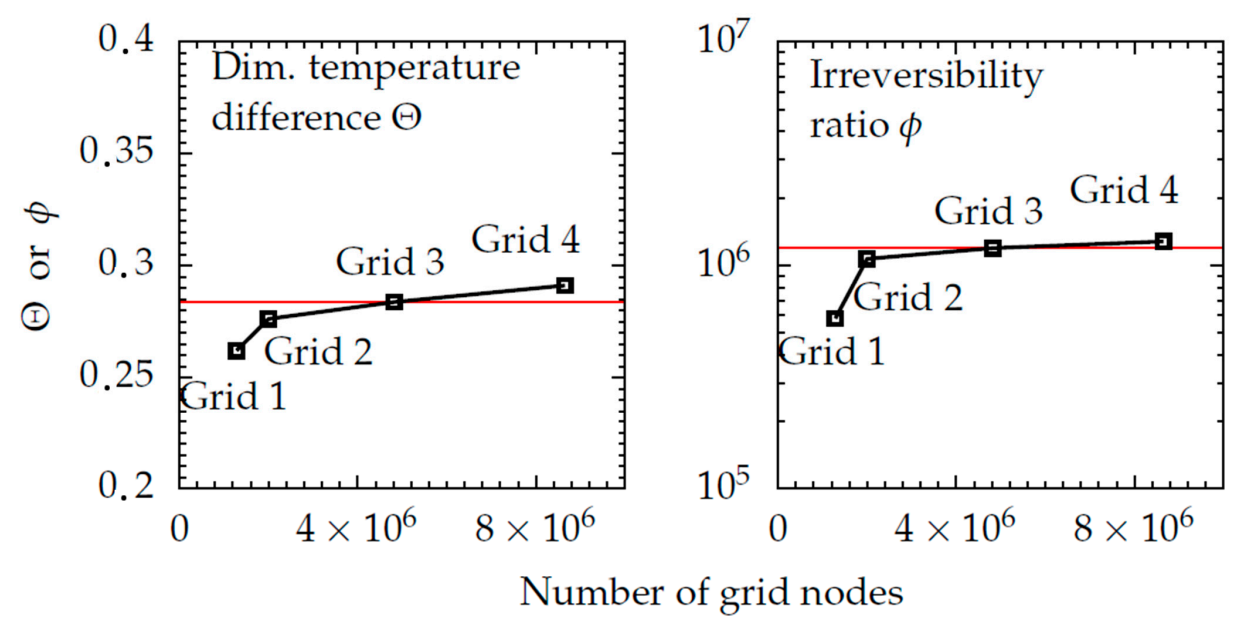

Figure 3. Grid-independence test for the V-Channel based on the dimensionless temperature difference (left) and the irreversibility ratio (right).

Here, $T_{W}$ is the area-averaged temperature of the cooling heat sink and $T_{\infty}$ is the ambient temperature. Only the smallest grid, grid 1 with $1.3 \times 10^{6}$ nodes, leads to a small but noticeable difference in $\Theta$ compared to the three other grids. Even more important to this study is the irreversibility ratio. The right hand side in Figure 3 shows the behavior of $\phi$ as a volume-integrated value over the whole V-Channel. Confirming the previous statement, the results of grid 1 differ strongly from those obtained for the three finer grids. Overall, grid 3 with $4.5 \times 10^{6}$ nodes (associated with the red lines in Figure 3) leads to a very good compromise between numerical effort and accuracy. Additionally, a dimensionless wall distance $y^{+} \approx 1$ is achieved for the heat sink wall with grid 3 . The relative deviations between grid 3 and the finest grid 4 with $8.7 \times 10^{6}$ nodes are only $2 \%$ for the dimensionless temperature and $6 \%$ for the irreversibility ratio. This level of accuracy is deemed sufficient in driving the optimization process, even more so when considering that optimization only requires correct predictions of trends, not of absolute values.

\section{Investigations of Heat Transfer}

In what follows, two different design modifications will be tested to intensify heat transfer. Based on the first analysis in Section 5.1, CFD-O will be used to optimize the position and alignment angle of two NACA profiles in Section 5.2. In Section 5.3, additional profiles will be manually included to increase the wetted area and hopefully enhance further heat transfer. To quantify the improvement, two values are systematically considered: (1) the temperature difference $\Delta T=T-T_{\infty}$ with $T$ as the volume-averaged temperature of the heat sink; and (2) the wall heat transfer coefficient $h=\dot{q} /\left(T_{W}-T_{\infty}\right)$ at the heat sink. Finally, Section 5.4 verifies the validity of the V-Channel results by comparison with a full 3D simulation of the alternator system.

\subsection{Base Design Analysis}

In this section, a classical analysis considering $T_{W}$ and $\mathbf{u}$ is first performed with the objective of manually finding regions with a high potential for optimization. A further analysis based on SLA uses the irreversibility ratio to identify such regions within the flow field. The following temperature and velocity scales are omitted for confidentiality reasons, since a real industrial design is considered. However, the employed ranges are constant, which enables a direct comparison between the following design changes.

Figure 4 shows the temperature distribution across the heat sink wall as well as the velocity vectors on the section plane introduced in Figure 1. 

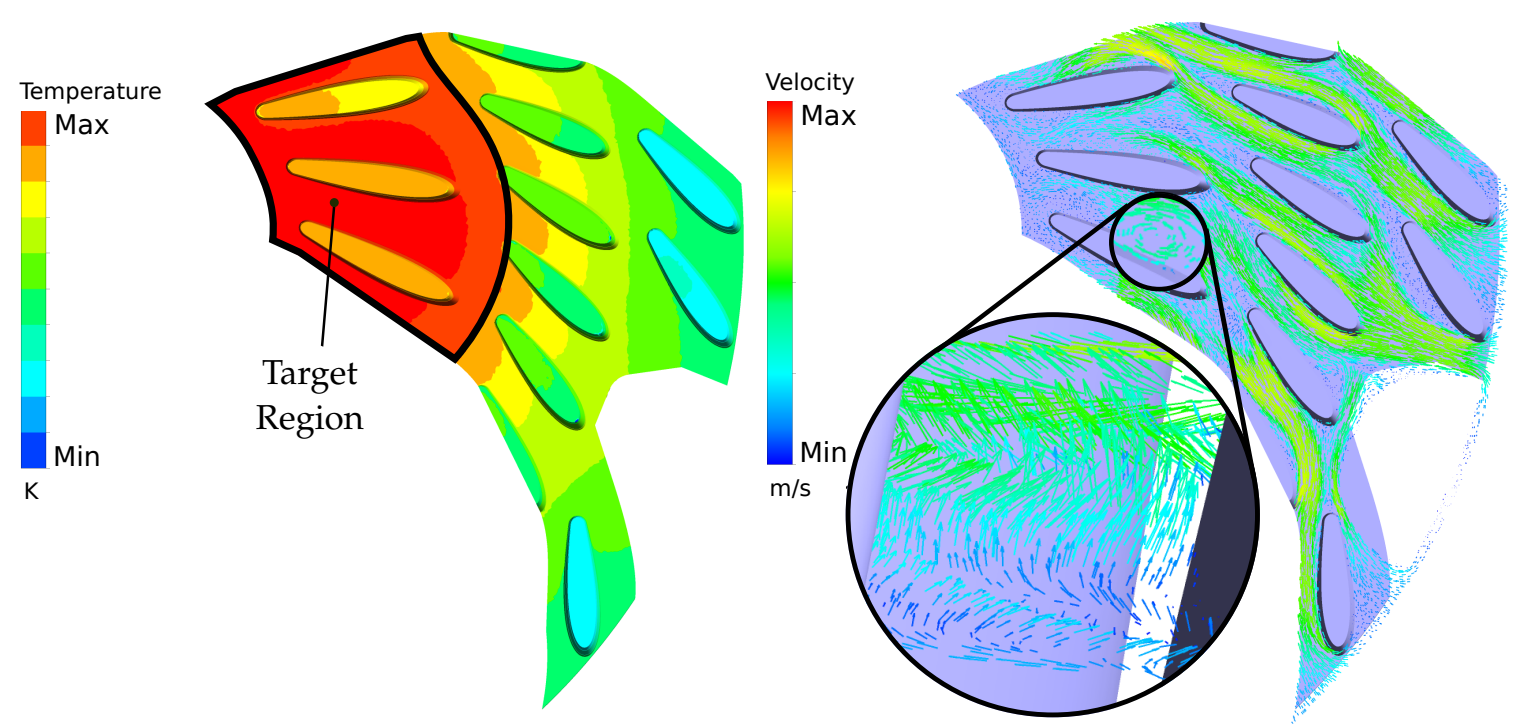

Figure 4. Classical analysis of heat transfer based on the temperature and the velocity field. The third row of profiles, as counted from the right (zoom), appears as a region with high improvement potential.

The figure shows high temperatures appearing in the left part of the domain. The electronic component is glued to the back side of the heat sink in this region. Additionally, the velocity field shows a back-flow region (see zoom in Figure 4). Therefore, optimizing the position of the profiles, or including additional exchange surfaces in this region seems to be a promising choice. Based on these first observations obtained by considering only the temperature and the velocity distributions, the NACA profiles at the farther end of the heat sink (third row from the right, see Figure 1) seem to have a high potential for optimization.

Figure 5 shows the irreversibility ratio for the same conditions.
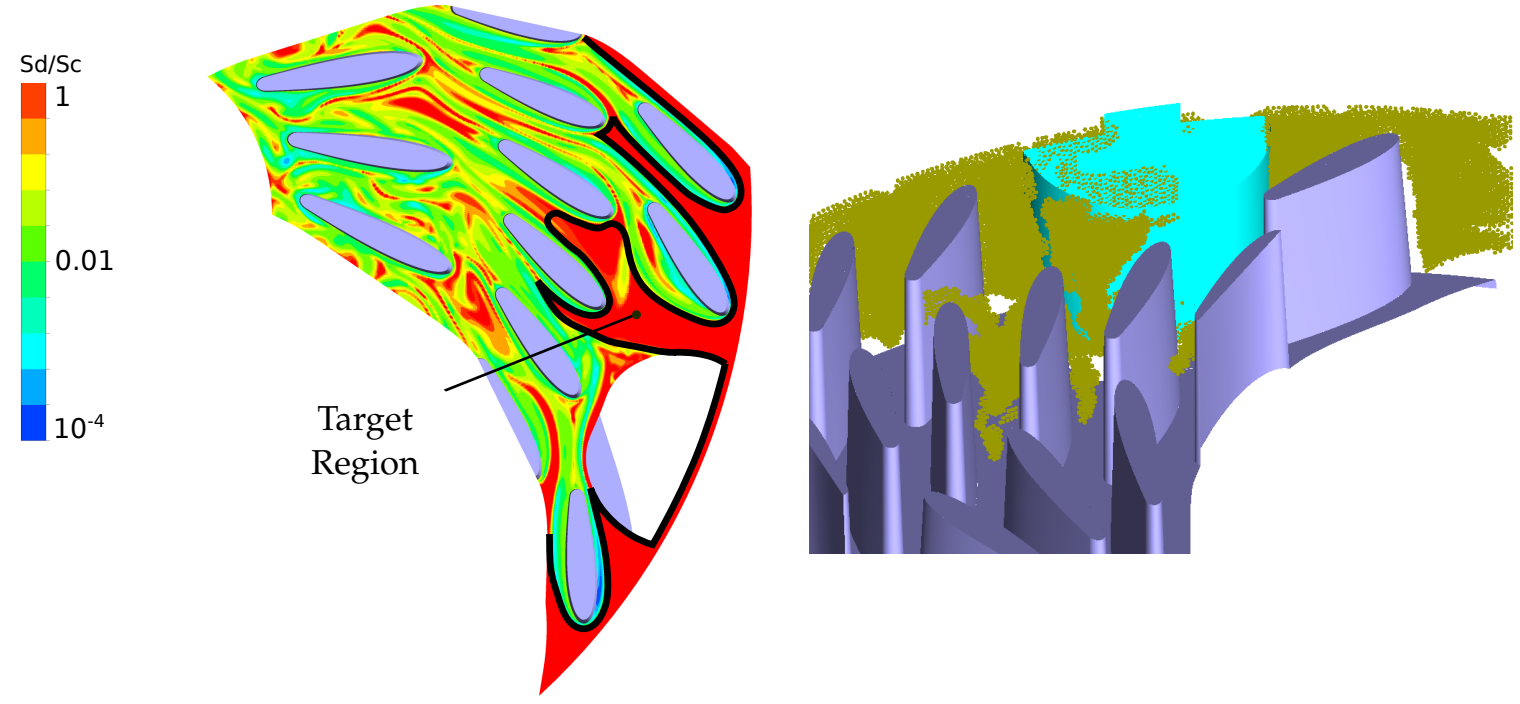

Figure 5. Advanced analysis based on the irreversibility ratio in the flow field. The left figure shows the same representation as Figure 4 . The right portion of the figure shows a representation of finite volumes with $\phi>1$ (dark yellow) from a different point of view, the third row now appearing at the bottom of this image. The blue area is part of the protection cap, shown in Figure 1.

Here, high magnitudes of the ratio between $\dot{S}_{D}^{\prime \prime \prime}$ and $\dot{S}_{C}^{\prime \prime \prime}$ occur mainly at the beginning of the heat sink (first two rows from the right, see Figure 1). According to our previous studies [3], these regions 
show a high potential for optimization. As shown in [3], if the velocity gradients (quantified indirectly by $\dot{S}_{D}^{\prime \prime \prime}$ ) are higher than the temperature gradients (quantified indirectly by $\dot{S}_{C}^{\prime \prime \prime}$ ), heat transfer can be improved locally. In the first row, very high values of $\phi$ are found in the complete flow field, as shown in Figure 5 (right). Here, every finite volume with $\phi>1$ is marked in dark yellow. It can be seen that, even for a three-dimensional field, the indicator $\phi>1$ can easily be post-processed and visualized in a complex geometry; it is a single, scalar value. Therefore, it appears to be an attractive indicator to guide optimization and is much easier to handle than a combination of temperature and velocity fields.

Now, the central question remains: observations based on classical indicators would result in an optimization at the farther end of the heat sink (third row from the right), since high temperatures and a back-flow area are found there, while SLA indicates that the first two rows on the right side have a high potential for improving heat transfer. Which one leads at the end to a higher heat transfer rate?

\subsection{Position Optimization}

The two central NACA profiles within the second heat sink row will now be optimized, as illustrated in Figure 6 (left).

These two profiles are located between both previously observed target regions shown in Figures 4 and 5, respectively. Both profiles can change their alignment angle in a range of $-20^{\circ}$ to $30^{\circ}$. Additionally, they can be moved along the chord, with an allowed displacement range of $-2 \mathrm{~mm}$ to $6 \mathrm{~mm}$. As a result, these NACA profiles are quite free to move within the region of high temperatures or high $\phi$-values, in a process driven by the employed optimizer. The OPtimization Algorithm Library++ (called OPAL++), developed at the Otto von Guericke University Magdeburg, Germany was used as an optimization tool. This software supports parallel execution and includes a variety of multi-objective as well as single-objective optimization algorithms. Further details can be found in $[32,33]$. For the present optimization, a simple single-objective genetic algorithm (called genetic1 in OPAL++) was applied. The minimization of the volume-averaged heat sink temperature was applied as an objective function. In this method,

- all variables have a real representation;

- each of the 50 generations contains 24 individuals;

- a tournament with two cycles is used to select parents;

- $\mathrm{SBX}$ is used for cross-over with a distribution index of $\eta_{c}=20$ and a probability of $p_{c}=0.8$ [34];

- a mutation with a distribution index of $\eta_{m}=10$ and probability of $p_{m}=1 / \#$ variables is applied, and

- the new generation replaces the old one.
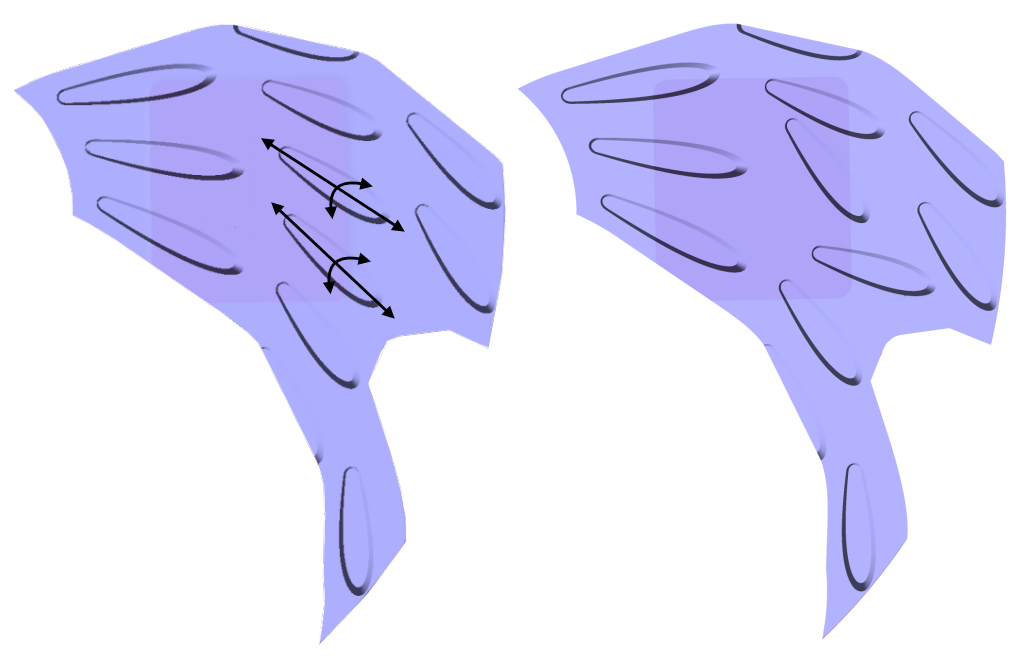

Figure 6. (Left): sketch illustrating the position optimization of the two central NACA profiles in the second row; (right): result of the optimization. 
For the computation, 3840 cores of a high-performance cluster were used, with 3.5 GB memory per core. A single CFD simulation takes about approximately $45 \mathrm{~min}$ to converge. During optimization, eight individuals are calculated in parallel, so that the total time needed for the optimization is approximately $900 \mathrm{~h}$.

Figure 7 shows the irreversibility ratio for the base design (left) and the optimized design (right), which are already found at the 35th generation of the genetic algorithm.
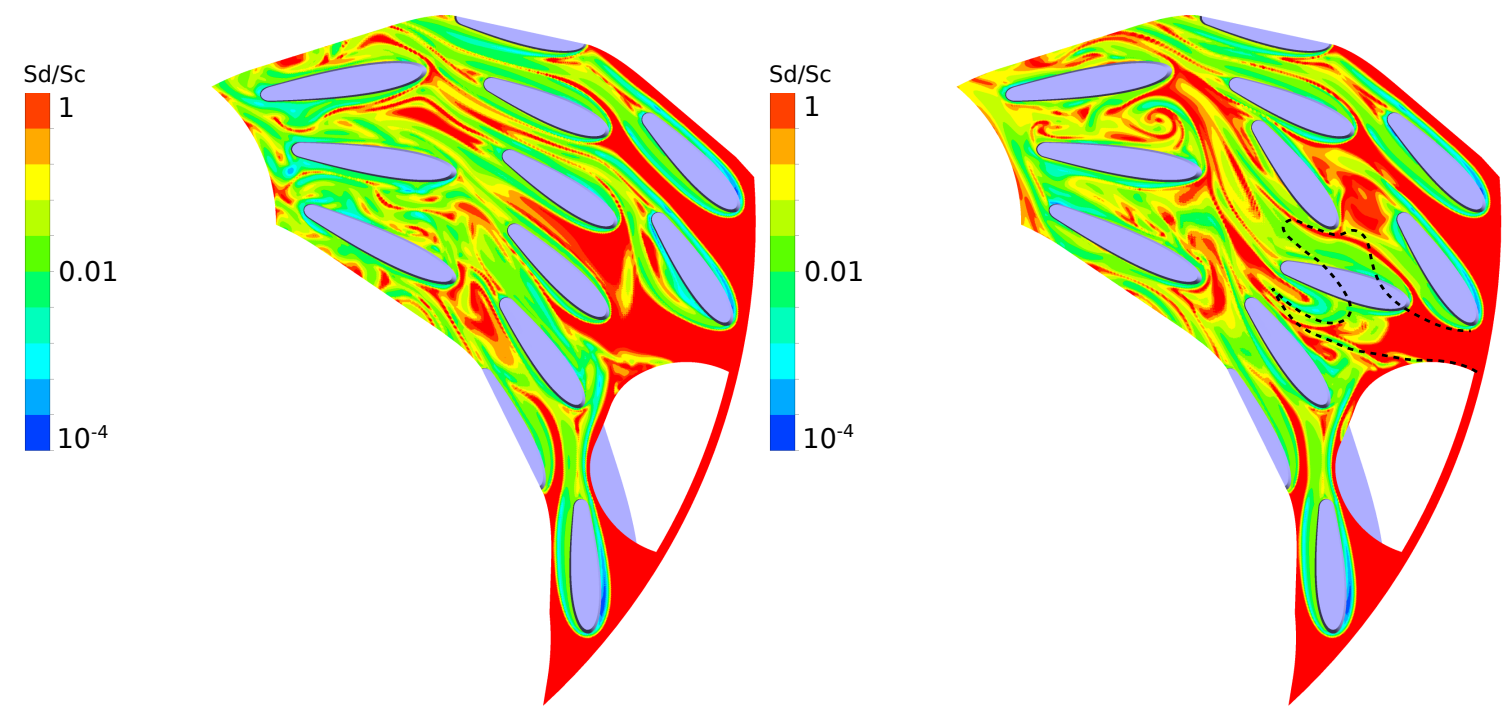

Figure 7. Optimized design resulting from the position optimization.

It can be seen that one NACA profile has moved forward to the right, towards the area with high irreversibility ratios, whereas the other one maintained its position but changed its angle. Neither of the two profiles moved back towards the third row, where high temperatures occur. The optimization direction chosen by OPAL++ is clear from the point of view of the SLA analysis (Figure 7): the region with large values of $\phi$ has been considerably reduced in size.

To quantify the improvement obtained by the optimization, Table 1 summarizes the results regarding the temperature difference and the wall heat transfer coefficient.

Table 1. Results of the position optimization, as difference between the optimized design and the baseline case.

\begin{tabular}{cc}
\hline $\begin{array}{c}\text { change in } \Delta T \\
\text { change in } h\end{array}$ & $-1.66 \mathrm{~K}(-4.83 \%)$ \\
\hline
\end{tabular}

The optimized design shows an improvement for both objective functions. Together with a wall heat transfer coefficient significantly increased by $6.46 \%$, the temperature difference from the heat sink to the fluid can be reduced by $-4.83 \%$, without including any additional surfaces, simply by moving and re-orientating the selected NACA profiles towards the region with a high irreversibility ratio. Now, increasing the exchange surface could perhaps present itself as a promising alternative.

\subsection{Increasing the Wetted Area}

Another possibility to improve heat exchange consists of introducing additional surfaces into the baseline design. Considering the two possibilities discussed at the end of Section 5.1, two additional NACA profiles with a scale ratio of 0.5 will be manually implemented: 
- $\quad$ near the third row, chosen after examining the temperature and velocity fields (Figure 8, left), or

- $\quad$ near the first row, considering the irreversibility ratio (Figure 8, right).

The obtained temperature fields are presented in Figure 8 as well.
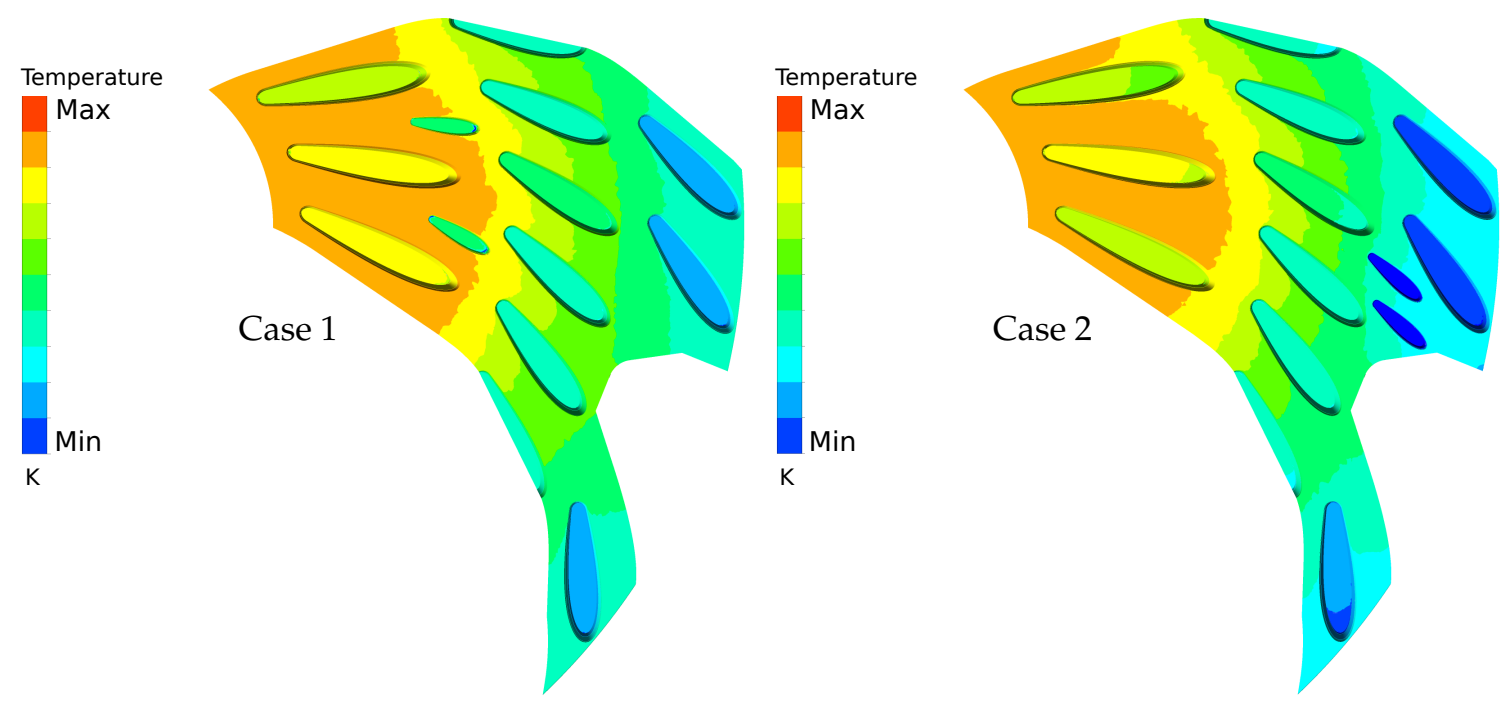

Figure 8. Temperature impact of two small additional NACA profiles introduced into the initially hot region (third row, left), or into the region with initially high $\phi$-values (first row, right). The color scale is identical for both figures.

Though this might initially appear counter-intuitive, it is clearly observed that the second solution (additional profiles in the first row, where the temperature is initially low) results in a lower temperature compared to the solution consisting of increasing the exchange surface in the hot region. The profiles in the first row are much cooler. Thus, heat transfer is intensified in this region. The other two rows, however, are also noticeably cooler than in the other configuration. The averaged values are summarized in Table 2.

Table 2. Results of placing small additional NACA profiles in either the third or the first row.

\begin{tabular}{lcc}
\hline & Third Row & First Row \\
\hline change in $\Delta T$ & $-1.42 \mathrm{~K}(-4.12 \%)$ & $-2.17 \mathrm{~K}(-6.30 \%)$ \\
change in $h$ & $-1.40 \mathrm{~W} / \mathrm{m}^{2} \mathrm{~K}(-2.29 \%)$ & $1.06 \mathrm{~W} / \mathrm{m}^{2} \mathrm{~K}(1.74 \%)$ \\
\hline
\end{tabular}

Considering these results in the view of practical purposes, the increasing pressure drop due to the additional NACA profiles in the first row seems to be negligible compared to the resulting, much better heat transfer. Both cases result in a lower cooling heat sink temperature, but the result is noticeably better for the modification obtained based on $\phi$. Concerning the heat transfer coefficient, while introducing additional profiles in the first row increases this value, as is desirable for the application, the opposite is observed for the alternative solution. Considering the definition of $h$,

$$
h=\frac{\dot{Q}}{A\left(T_{W}-T_{\infty}\right)},
$$

the wall heat transfer coefficient directly combines the increasing surface area $A$ with the decreasing temperature difference on the surface wall $\Delta T$. For the present conditions ( $\dot{Q}=$ const. and $T_{\infty}=$ const.), the unwanted decrease of $h$ when adding profiles in the third row is the result of a lower benefit (decreasing $T_{W}$ ) compared to a higher cost (increasing $A$ ). Considering these results, it appears that it is not always beneficial to add supplementary exchange surfaces in hot-temperature regions, since 
they might have a noticeably lower effect on the heat transfer rate, even if a simpler analysis based on temperature could suggest such modifications. Analyzing the irreversibility ratio seems to be a far more promising solution. Based on such an analysis of $\phi$, regions within the flow field that possess a high potential for heat transfer optimization can easily be located, leading to a tremendous reduction of the required number of design parameters and of the size of the parameter space. In this way, optimizing the alternator with CFD-O becomes possible.

A last possible design modification is shown in Figure 9.
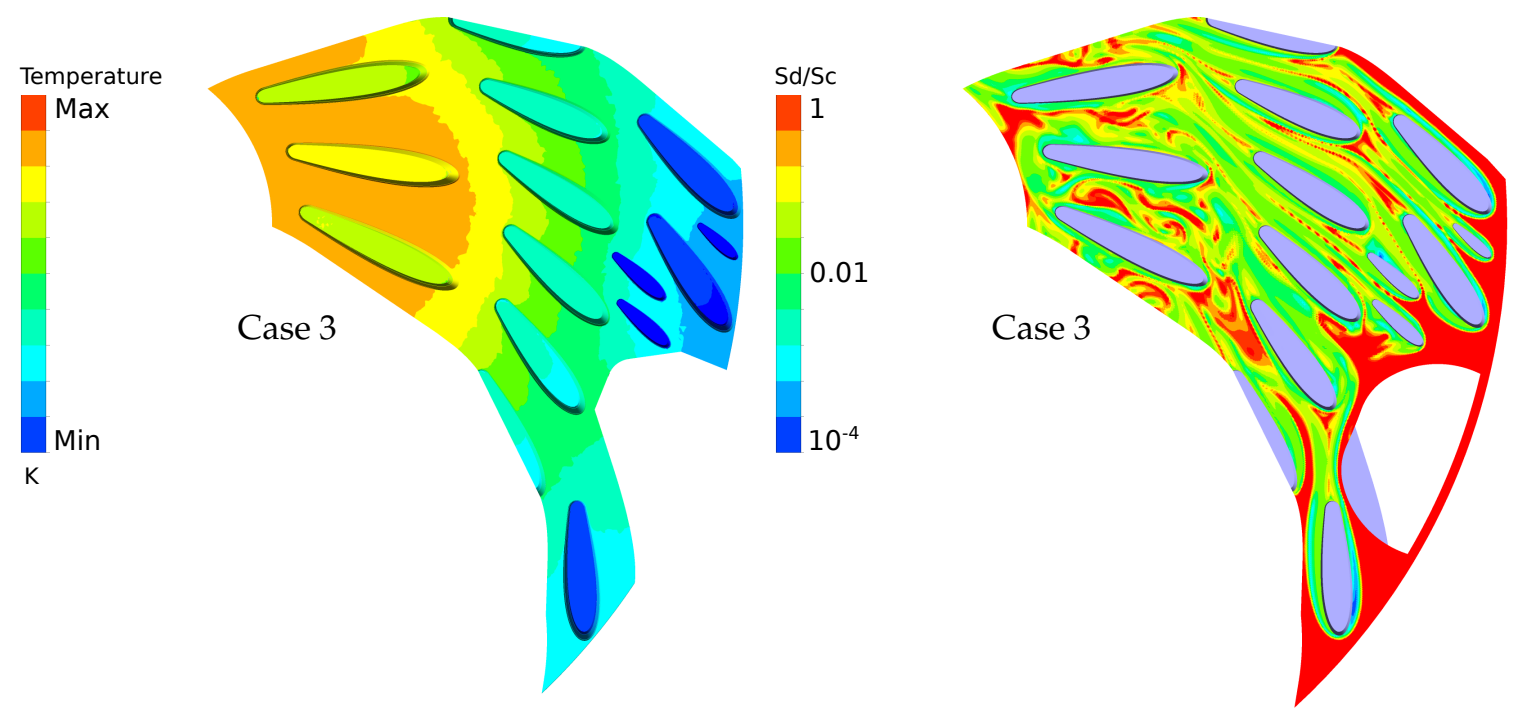

Figure 9. Adding three small NACA profiles to increase heat transfer.

Here, a third NACA profile (scale ratio $=0.25$ ) has been manually placed in the first row, on the inflow side of the heat sink. As illustrated, all three profiles are placed directly in the region where values of $\phi>1$ initially appear. This modification further increases the heat transfer and thus results in the lowest temperature difference obtained during this study. Table 3 summarizes the obtained progress compared to the original design.

Table 3. Results of the design modification when adding three small NACA profiles.

\begin{tabular}{cc}
\hline change in $\Delta T$ & $-2.31 \mathrm{~K}(-6.72 \%)$ \\
change in $h$ & $0.74 \mathrm{~W} / \mathrm{m}^{2} \mathrm{~K}(1.22 \%)$ \\
\hline
\end{tabular}

Compared to the results obtained by optimizing the position of the original NACA profiles with OPAL++, the obtained enhancement of the heat transfer coefficient is smaller (1.22\% in this case), yet leads to the lowest heat sink temperature $(-6.72 \%)$.

\subsection{Comparison with Full Model}

All the results in the previous section were solely obtained for the V-channel. It is important to verify that they would also be valid for the full alternator system, using the same workflow. In this case, the pressure drop resulting from the rotating fans is directly simulated. No model is needed to approximate the operating point.

Figure 10 shows the irreversibility ratio along the section plane, located at mid-height of the NACA profiles within the full model. 

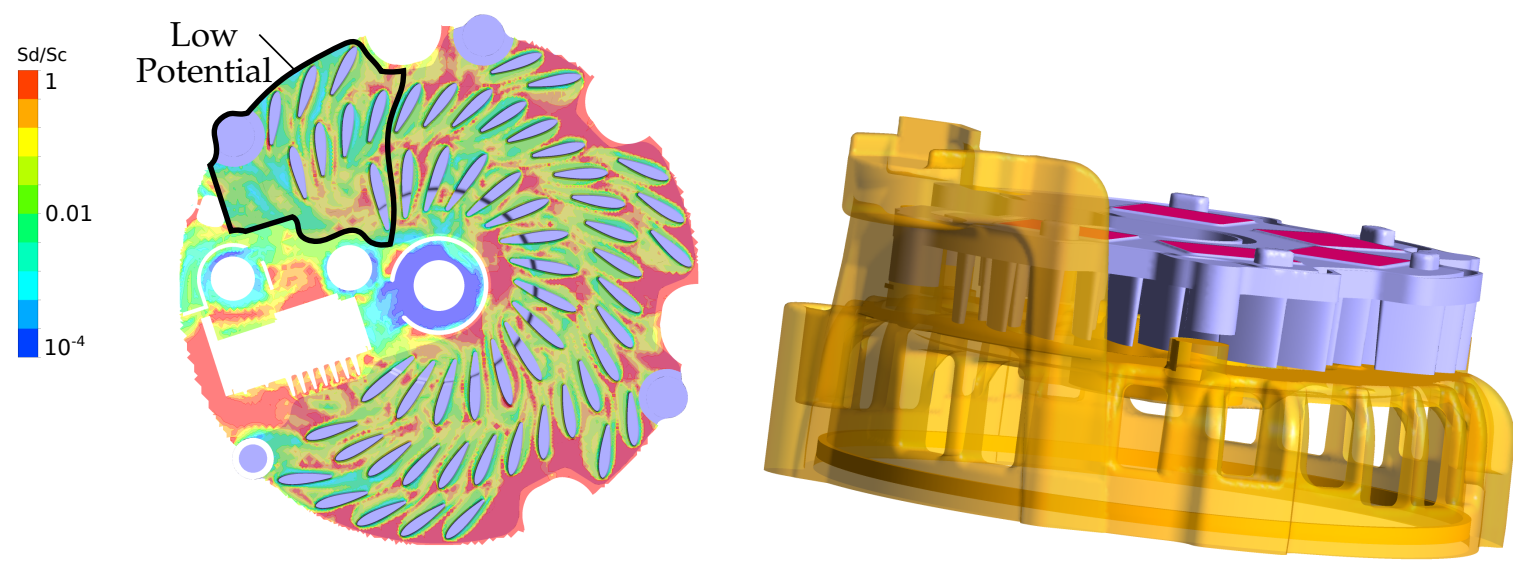

Figure 10. (Left): irreversibility ratio along a section plane in the full alternator model; (right): retention arm passing in the axial direction along the heat sink. The flow passes over the heat sink from the outer radial direction.

As in the V-Channel, regions with high $\phi$-values occur near the inflow side of the heat sink, except in the highlighted area contained within the thick black line. Here, most of the values have a magnitude of $\phi<1$ and are thus not interesting for optimization. The reason for this effect is found on the right-hand side in Figure 10. In the full model, the retention arm of the B-endshield is present. To ensure the connection to the motor, the retention arm is made of a solid material; therefore, it prevents fluid from streaming through the nearby NACA profiles. A design modification in this region would have a much smaller effect on heat transfer compared to the other inflow sides. Nevertheless, in order to maintain the symmetry of the setup and to increase the wetted area, additional profiles will be placed in this region as well.

Figure 11 shows the temperature fields in the original and in the improved design.
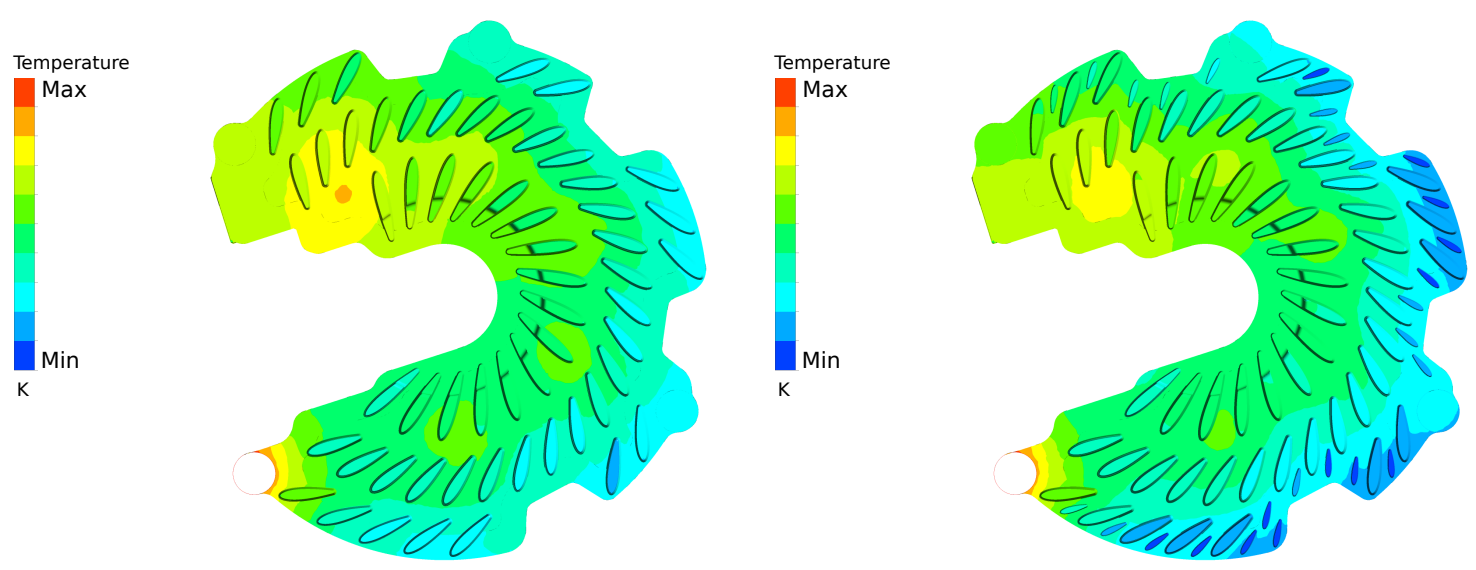

Figure 11. Enhancing heat transfer in an electric machine. Comparison of the local temperature distribution in the original design (left) and in the improved design after SLA (right).

As in the previous V-Channel study, the temperature in the inflow side becomes noticeably lower compared to its original value. The original design shows a high temperature peak behind the retention arm, due to the limited flow rate leading to low cooling performance; this problem is much less pronounced in the improved design. 
Thanks to the manual design modifications driven by the SLA, reduced temperatures are found across the complete heat sink; a more homogeneous temperature distribution is obtained. Table 4 summarizes the enhancements obtained by adding 27 small NACA profiles.

Table 4. Results of the design improvement for the full model.

\begin{tabular}{cc}
\hline $\begin{array}{c}\text { change in } \Delta T \\
\text { change in } h\end{array}$ & $-2.00 \mathrm{~K}(-6.43 \%)$ \\
\hline
\end{tabular}

The positive trend observed for the V-Channel is confirmed by this full-model study. The observed improvements concerning $\Delta T$ are nearly identical in both cases. While the V-Channel indicated an enhancement of $-2.31 \mathrm{~K}(-6.72 \%)$, the full model leads to a reduction of $-2.00 \mathrm{~K}(-6.43 \%)$, which is still considerable for practical purposes. Larger discrepancies between the V-Channel and the full model simulation are observed for the wall heat transfer coefficient. The positive effect found in the V-Channel (increase of $0.74 \mathrm{~W} / \mathrm{m}^{2} \mathrm{~K}$, or $1.22 \%$ ) is reduced in the full model simulation, with only $0.40 \mathrm{~W} / \mathrm{m}^{2} \mathrm{~K}(0.64 \%)$. It should be kept in mind that both models are not identical, due to the periodic assumption employed for the V-Channel. The heat sink is considered as a closed part for the V-Channel, but not in the full model (see Figure 11). In the full model, the position and the amount of inserted NACA profiles differ from the retained configuration in the V-channel. Therefore, the results can not be directly compared with one another.

However, both simulations (V-Channel and full model) show the same, positive trend for both objective functions, minimizing the temperature difference $\Delta T$ and maximizing the heat transfer coefficient $h$.

\section{Conclusions}

Based on the fundamental analysis with the canonical configuration in [3], a powerful concept has been applied in this work to analyze heat transfer processes and ultimately optimize practical cooling systems. This has been demonstrated for a real alternator heat sink. Physical values such as temperature or wall heat transfer coefficient (classical indicators) can, in principle, be used as an objective function to quantify the intensity of heat transfer. However, they convey no further information on the details of the complex transport phenomena occurring within the flow field. To compare different transport processes and to find regions where a design modification could lead to an intensified heat transfer, further indicators based on the second law of thermodynamics are more useful. General investigations of heat transfer based on the canonical configuration in [3] and the presented application to a heat sink of an electric machine have shown that the irreversibility ratio is particularly promising for this purpose. Being a scalar quantity and containing information on both convection and diffusion, $\phi$ offers a very convenient method to analyze a complex three-dimensional flow field in an easy and efficient manner, much more so than by considering the velocity and temperature distributions separately. Based on this analysis, optimizing regions with $\phi>1$ leads to better designs than those obtained from a classical analysis derived from temperature and velocity. Based on the irreversibility ratio, CFD-based optimization becomes practical, since the associated numerical effort is highly reduced by concentrating only on key regions of the device.

This work confirms the preliminary findings obtained for the canonical configuration in [3], showing that this simple problem can be used for testing alternative approaches. Further investigations based on the irreversibility ratio will now consider further technical applications.

Additionally, the same analysis can be applied globally to the complete alternator system, as exemplified in Figure 12. 

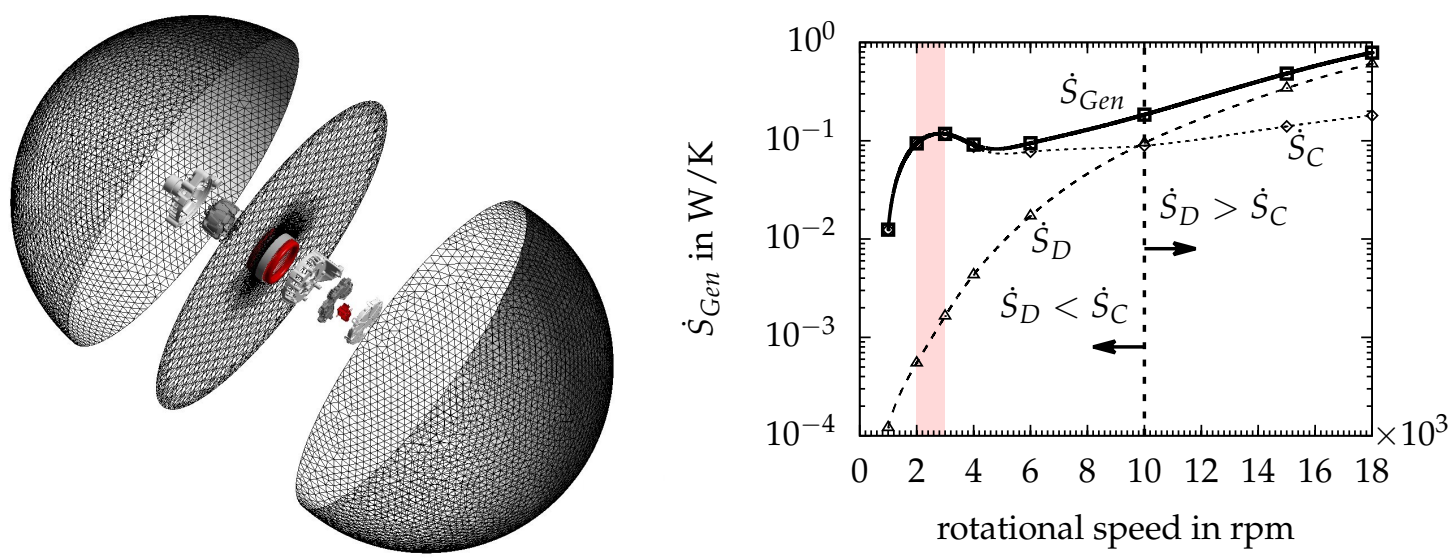

Figure 12. (Left): explosion view of the alternator system and the modeled spherical ambient defined with an opening boundary condition (constant pressure and temperature). The diameter of the ambient sphere is more than six times greater than the alternator diameter; (right): entropy generation terms computed for a full alternator system as a function of rotation speed [35]. The red region corresponds to that with the highest temperature of the electronic components, between $2000 \mathrm{rpm}$ to $3000 \mathrm{rpm}$.

Here, the volume integral of each entropy generation term $\left(\dot{S}_{C}\right.$ and $\left.\dot{S}_{D}\right)$ has been calculated within the complete spherical region shown in Figure 12. The examined alternator system is based on another electrical concept and can therefore not be compared with the alternator designs investigated before. It can be seen that, for the range of $1000 \mathrm{rpm}$ to $10,000 \mathrm{rpm}$, entropy generation due to conduction $\left(\dot{S}_{C}\right)$ is the dominating process and reaches its local maxima between $2000 \mathrm{rpm}$ to $3000 \mathrm{rpm}$. The behavior of $\dot{S}_{C}$ is directly correlated to the volume averaged temperature for electronic components such as cooling heat sinks or rectifiers where its maximum temperature $T_{\max }$ is within the same range of $2000 \mathrm{rpm}$ to $3000 \mathrm{rpm}$ (marked in red in Figure 12). Due to a high electrical current and a low volume flow in this range, the maximum temperature as well as entropy generation due to conduction occurs at approximately $3000 \mathrm{rpm}$. This confirms the correlation between entropy generation due to conduction $\dot{S}_{C}$ and the component temperature $T$ as already investigated in [2]. Increasing the rotational speed above 10,000 rpm causes entropy generation due to dissipation to become the dominating factor; at this point, total entropy generation is dominated by flow convection. Based on such an analysis, operation and regulation of the system could be analyzed and optimized. This also offers a comparison between different cooling designs on a global level.

Author Contributions: All authors contributed to the research, whereas Toni Eger wrote the main paper. All authors discussed the results and implications and commented on the manuscript at all stages. All authors have read and approved the final manuscript.

Conflicts of Interest: The authors declare no conflict of interest.

\section{Nomenclature}

\section{Roman Letters}

$\dot{S}_{C} \quad$ Entropy generation due to conduction

$\dot{S}_{D} \quad$ Entropy generation due to dissipation

$\dot{S}_{\text {Gen }}$ Total entropy generation

$h \quad$ Wall heat transfer coefficient

$k$ Thermal conductivity

$s_{12} \quad$ First node distance

$s_{\max }$ Maximum element size

$T_{W} \quad$ Wall temperature 


\section{Greek Letters}

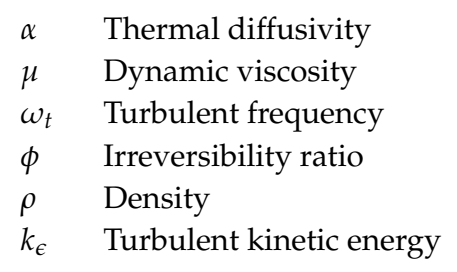

\section{Dimensionless Quantities}

$\mathrm{Pr}_{t} \quad$ Turbulent Prandtl number

$\Theta \quad$ Dimensionless temperature difference

$y^{+} \quad$ Dimensionless wall distance

\section{Superscripts}

Time mean component

Fluctuation component

Value per volume

\section{References}

1. Thévenin, D.; Janiga, G. Optimization and Computational Fluid Dynamics; Springer: Berlin, Germany, 2008.

2. Eger, T.; Bol, T.; Thévenin, D.; Schroth, R.; Janiga, G. Preliminary numerical investigations of entropy generation in electric machines based on a canonical configuration. Entropy 2015, 17, 8187-8206.

3. Eger, T.; Bol, T.; Daróczy, L.; Janiga, G.; Schroth, R.; Thévenin, D. Numerical investigations of entropy generation to analyze and improve heat transfer processes in electric machines. Int. J. Heat Mass Transf. 2016, 102, 1199-1208.

4. Eger, T.; Thévenin, D.; Janiga, G.; Bol, T.; Schroth, R. Identification of a Canonical Configuration for a Quantitative Analysis of Transport Phenomena in Electric Machines Based on Entropy Generation. In Energy and Sustainability V; Al-Kayiem, H., Brebbia, C., Zubir, S., Eds.; WIT Press: Southampton, UK, 2014; Volume 186, pp. 751-762.

5. Eger, T.; Thévenin, D.; Janiga, G.; Bol, T.; Schroth, R. Numerical investigations of residence time distribution of air in electric machines based on a canonical configuration. In Proceedings of the Conference on Modelling Fluid Flow, Budapest, Hungary, 1-4 September 2015; pp. 1-8.

6. Bejan, A. Entropy generation minimization: The new thermodynamics of finite size devices and finite time processes. J. Appl. Phys. 1996, 79, 1191-1218.

7. Bejan, A. Method of entropy generation minimization, or modeling and optimization based on combined heat transfer and thermodynamics. Revue Générale de Thermique 1996, 35, 637-646.

8. Bejan, A.; Lorente, S. The constructal law and the evolution of design in nature. Phys. Life Rev. 2011, 8, 209-240.

9. Poulikakos, A.; Bejan, A. Fin geometry for minimum entropy generation in forced convection. J. Heat Transf. 1982, 104, 616-623.

10. Fowler, A.J.; Bejan, A. Correlation of optimal sizes of bodies with external forced convection heat transfer. Int. Commun. Heat Mass Transf. 1994, 21, 17-27.

11. Carrington, C.; Sun, Z. Second law analysis of combined heat and mass transfer in internal and external flows. Int. J. Heat Fluid Flow 1992, 13, 65-70.

12. Ko, T.; Ting, K. Entropy generation and optimal analysis for laminar forced convection in curved rectangular ducts: A numerical study. Int. J. Therm. Sci. 2006, 45, 138-150.

13. Şahin, A.Z. Irreversibilities in various duct geometries with constant wall heat flux and laminar flow. Energy 1998, 23, 465-473.

14. Chen, C.T.; Chen, H.I. Multi-objective optimization design of plate-fin heat sinks using a direction-based genetic algorithm. J. Taiwan Inst. Chem. Eng. 2013, 44, 257-265.

15. Adham, A.M.; Mohd-Ghazali, N.; Ahmad, R. Optimization of a rectangular microchannel heat sink using entropy generation minimization (EGM) and genetic algorithm (GA). Arab. J. Sci. Eng. 2014, 39, 7211-7222.

16. Herwig, H.; Wenterodt, T. Second law analysis of momentum and heat transfer in unit operations. Int. J. Heat Mass Transf. 2011, 54, 1323-1330. 
17. Jin, Y.; Herwig, H. Turbulent flow and heat transfer in channels with shark skin surfaces: Entropy generation and its physical significance. Int. J. Heat Mass Transf. 2014, 70, 10-22.

18. Wenterodt, T.; Herwig, H. The entropic potential concept: A new way to look at energy transfer operations. Entropy 2014, 16, 2071-2084.

19. Kock, F.; Herwig, H. Local entropy production in turbulent shear flows: A high-Reynolds number model with wall functions. Int. J. Heat Mass Transf. 2004, 47, 2205-2215.

20. Kock, F.; Herwig, H. Entropy production calculation for turbulent shear flows and their implementation in CFD codes. Int. J. Heat Fluid Flow 2005, 26, 672-680.

21. Giangaspero, G.; Sciubba, E. Application of the EGM method to a LED-based spotlight: A constrained pseudo-optimization design process based on the analysis of the local entropy generation maps. Entropy 2011, 13, 1212-1228.

22. Giangaspero, G.; Sciubba, E. Application of the entropy generation minimization method to a solar heat exchanger: A pseudo-optimization design process based on the analysis of the local entropy generation maps. Energy 2013, 58, 52-65.

23. Khan, W.; Yovanovich, M.; Culham, J. Optimization of microchannel heat sinks using entropy generation minimization method. In Proceedings of the Semiconductor Thermal Measurement and Management Symposium, Dallas, TX, USA, 14-16 March 2006; pp. 78-86.

24. Yang, W.J.; Furukawa, T.; Torii, S. Optimal package design of stacks of convection-cooled printed circuit boards using entropy generation minimization method. Int. J. Heat Mass Transf. 2008, 51, 4038-4046.

25. Shih, C.; Liu, G. Optimal design methodology of plate-fin heat sinks for electronic cooling using entropy generation strategy. IEEE Trans. Compon. Packag. Technol. 2004, 27, 551-559.

26. Culham, J.; Muzychka, Y. Optimization of plate fin heat sinks using entropy generation minimization. IEEE Trans. Compon. Packag. Technol. 2001, 24, 159-165.

27. Sciacovelli, A.; Verda, V.; Sciubba, E. Entropy generation analysis as a design tool-A review. Renew. Sustain. Energy Rev. 2015, 43, 1167-1181.

28. Kock, F. Bestimmung der Lokalen Entropieproduktion in Turbulenten Strömungen und deren Nutzung zur Bewertung Konvektiver Transportprozesse. Ph.D. Thesis, Technische Universität Hamburg-Harburg, Hamburg, Germany, 2003. (In German)

29. Bejan, A. Entropy Generation Minimization: The Method of Thermodynamic Optimization of Finite-Size Systems and Finite-Time Processes. In Mechanical and Aerospace Engineering Series; CRC Press: Florida, FL, USA, 1995.

30. Gesellschaft, V. VDI Heat Atlas; Springer: Berlin, Germany, 2010.

31. Menter, F. Improved Two-Equation k-Mmega Turbulence Models for Aerodynamic Flows; Technical Report; NASA Ames Research Center: Moffett Field, CA, USA, 2008.

32. Daróczy, L.; Janiga, G.; Thévenin, D. Systematic analysis of the heat exchanger arrangement problem using multi-objective genetic optimization. Energy 2014, 65, 364-373.

33. Daróczy, L. Practical Issues in the Optimization of CFD Based Engineering Problems. Ph.D. Thesis, Otto von Guericke University Magdeburg, Magdeburg, Germany, 2016. Available online: http:/ / edoc2.bibliothek.unihalle.de/hs/content/titleinfo/61051 (accessed on 29 May 2017).

34. Deb, K.; Agrawal, R.B. Simulated binary crossover for continuous search space. Complex Syst. 1995, 9, 115-148.

35. Shi, K. Integrale Analyse der Wärmeübertragung in Elektrischen Generatoren. Master's Thesis, Karlsruhe Institute of Technology, Karlsruhe, Germany, 2016. (In German)

(c) 2017 by the authors. Licensee MDPI, Basel, Switzerland. This article is an open access article distributed under the terms and conditions of the Creative Commons Attribution (CC BY) license (http://creativecommons.org/licenses/by/4.0/). 